\title{
Feasibility of Computational Fluid Dynamics for Evaluating the Intraventricular Hemodynamics in Single Right Ventricle Based on Echocardiographic Images
}

\author{
Li-Jun Chen $\mathbb{D}^{1}{ }^{1}$ Zhi-Rong Tong, ${ }^{2,3}$ Qian Wang, ${ }^{4}$ Yu-Qi Zhang $\mathbb{D}^{1},{ }^{1}$ and Jin-Long Liu $\mathbb{D}^{2,3}$ \\ ${ }^{1}$ Department of Pediatric Cardiology, Shanghai Children's Medical Center, Shanghai Jiaotong University School of Medicine, \\ Shanghai 200127, China \\ ${ }^{2}$ Department of Cardiothoracic Surgery, Shanghai Children's Medical Center, Shanghai Jiaotong University School of Medicine, \\ Shanghai 200127, China \\ ${ }^{3}$ Institute of Pediatric Translational Medicine, Shanghai Children's Medical Center, Shanghai Jiaotong University School of Medicine, \\ Shanghai 200127, China \\ ${ }^{4}$ Department of Medical Imaging, Shanghai Children's Medical Center, Shanghai Jiaotong University School of Medicine, \\ Shanghai 200127, China
}

Correspondence should be addressed to Yu-Qi Zhang; zhangyuqi6812@163.com

Received 16 August 2017; Revised 6 December 2017; Accepted 11 December 2017; Published 16 January 2018

Academic Editor: Hwa-Liang Leo

Copyright (C) 2018 Li-Jun Chen et al. This is an open access article distributed under the Creative Commons Attribution License, which permits unrestricted use, distribution, and reproduction in any medium, provided the original work is properly cited.

\begin{abstract}
This study introduced a combined computational fluid dynamics (CFD) and echocardiography methodology to simulate blood flow in the single right ventricle (SRV) and normal ventricles to study the intraventricular flow. Derived from echocardiographic image loops, CFD-based three-dimensional (3D) flow models of normal subject's left ventricle (LV) and right ventricle (RV) and SRV with and without heart failure at three characteristic diastolic statuses were reconstructed. The CFD derived morphological and functional measurements in normal ventricles and the SRV were validated with echocardiography. The vortex in the normal ventricles and the SRV were studied. The morphological and functional measurements derived from CFD modeling and echocardiography were comparable, and both methods demonstrated the larger volume and higher spherical index in the SRV, in particular the SRV with heart failure. All the vortices in the SRV were smaller than those in the normal control subject's LV and RV, notably with heart failure. Unlike normal LV and RV, no vortex ring was observed in the SRV. Echocardiography-based CFD demonstrated the feasibility of quantifying ventricular morphology and function; in addition, CFD can detect the abnormal flow pattern (smaller or obliterated vortices) in the SRV when compared with normal ventricles.
\end{abstract}

\section{Introduction}

Single right ventricle (SRV) is a congenital heart disease which causes severe cyanosis. SRV exhibits a large right ventricle as the dominant ventricular chamber (DVC) and a hypoplastic left ventricle as the rudimentary chamber (RC). The SRV has the right ventricle anatomy but works as both pulmonic and systemic circulation ventricles; it is usually unable to sustain separate pulmonary and systemic circulations in sequence, nevertheless offering normal ventricular performance. The SRV has impaired systolic and diastolic function compared with that of the respective ventricles in a normal biventricular heart [1].

Systolic dysfunction of the ventricle accompanied with abnormal diastolic function can be gauged by the ventricular filling impairment [2]. The Working Group for the European Society of Cardiology proposed that diagnosis of diastolic heart failure requires not only presence of clinical signs or symptoms, but also evidence of abnormal filling [3]. Visualization of the flow pattern inside the ventricle during filling phase provides incremental value of understanding the flow dynamics; it also allows better insights into both 
TABLE 1: Basic demographic information and clinical characteristics of the study subjects.

\begin{tabular}{lccc}
\hline & Control & SRV & SRV + HF \\
\hline Age $(\mathrm{y})$ & 4 & 4 & 4 \\
Gender & Male & Male & Male \\
Height $(\mathrm{cm})$ & 100 & 101 & 107 \\
Weight $(\mathrm{Kg})$ & 15.0 & 15.0 & 19.0 \\
BSA $\left(\mathrm{m}^{2}\right)$ & 0.65 & 0.65 & 0.75 \\
HR (beat $/ \mathrm{min})$ & 118 & 92 & 90 \\
BP $(\mathrm{mmHg})$ & $98 / 56$ & $108 / 58$ & $100 / 63$ \\
\hline
\end{tabular}

Note. BSA: body surface area; HR: heart rate; BP: blood pressure; SRV: single right ventricle without heart failure; SRV + HF: single right ventricle with heart failure.

physiologic and pathophysiologic process during the cardiac cycle. Analysis of the properties of ventricular flow can prompt early detection of cardiac dysfunctions $[4,5]$.

Four-dimensional flow cardiac magnetic resonance (CMR) can visualize intracardiac flow characteristics in the heart and vessels [6], but the poor temporal resolution of CMR only allows flow visualization based on the averaged flow signals over several cardiac cycles [6], which cannot provide real-time dynamic flow information. Vector flow mapping (VFM) and Echo particle imaging velocimetry (EPIV) both are based on echocardiography (Echo) images and allow visualization of flow in the left ventricle $[7,8]$, but the inherently low temporal and spatial resolution from Echo limit their uses. Cardiac catheterization and radionuclide angiography were not routinely used to visualize the cardiac flow due to radiation.

Computational fluid dynamics (CFD) can investigate blood flow dynamics in the vessel as well as in the ventricle and atrium $[9,10]$. CFD can analyze blood patterns in any heart with any anatomic abnormalities due to no limitations of geometry assumption. To our knowledge, this is the first study on intraventricular flow of single right ventricle using CFD.

This study aimed to provide a primary step in the numerical simulation of fluid dynamics in a SRV with and without heart failure and to compare them with those of normal left and right ventricle using CFD, in particular, to analyze vortices during diastole to provide detailed information for physicians to give patients timely intervention as soon as possible.

\section{Methods}

The study was performed on a male subject who was diagnosed with SRV at two stages (with and without heart failure), and an age- and gender-matched normal child (Table 1). The parents of the study children gave informed consent. The study design, manner of data collection, and analysis were approved by the local institutional review board (IRB) and regional research ethics committee (REC) of Shanghai Children's Medical Center Affiliated to Shanghai Jiaotong University School of Medicine. All the methods in this study were performed in accordance with the IRB and REC guidelines and regulations.
Full-volume, three-dimensional (3D) echocardiographic image loops of the heart were obtained by an IE33 ultrasound machine (Philips, Andover, MA, USA) equipped with a $3 \mathrm{D}$ transducer (X5-1). The transducer frequency $(1.6-3.2 \mathrm{MHz})$ was optimized for image acquisition. The image depth $(10 \mathrm{~cm})$ and sector width $\left(110^{\circ}\right)$ were adjusted to maximize the frame rate ( $>30$ frames/second). The echo image loops were used to reconstruct the chamber geometry and then for further simulations. Echo image segmentation was achieved by using medical imaging software (Materialise ${ }^{\circledR}$-Mimics 17.5, Plymouth, MI, USA) and then the 3D ventricular model was reconstructed (Figure 1). Initially, the full-volume echo images were imported into the software; then a "Thresholding" tool was applied to specify the range of the ventricular wall. After defining the ventricular wall, a "Multiple Slice Edit" tool was applied to edit multiplane images to remove the surrounding noncardiac structures. An addition "Edit Masks" tool will be employed if the automatic identification for the cardiac anatomy was unclear. The "Region Growing" tool was then used to generate the $3 \mathrm{D}$ ventricular model; once the model was established the "Smooth Mask" tool was used to smooth the 3D model for subsequent simulations. The papillary muscles and valves for the $3 \mathrm{D}$ model were smoothed out.

In addition to the full-volume 3D echo image loops, $2 \mathrm{D}$ echo image loops were acquired with a S5-1 transducer at $>60$ frames per second. The pulse wave Doppler was used to determine the flow curve at the inlet and outlet of LV (Figure 2, Table 2). The velocities of the inlet and the outlets of the LV were imported into the software (ANSYS-FLUENT 17.0) as the boundary conditions for CFD simulations.

The cardiac anatomy was determined by echocardiography and then represented by five body-fitted prism layers as boundaries (ANSYS-ICEM 17.0) for numerical solutions of the Navier-Stokes flow equations. The distance from the first layers to the model surface was set as $0.02 \mathrm{~mm}$. A tetrahedral mesh, containing approximately $1,000,000$ to $3,000,000$ tetrahedral mesh elements, was used to fill the remainder of the calculated domain. The computational domain was extended to include the proximal atrium and ascending aorta or pulmonary artery for accurate simulation and calculation. The simulation was accomplished by using the ventricular geometry for reconstruction and the velocities of inflow/outflow as boundary conditions. Finally, the visualization of streamlines was completed by the ANSYS 


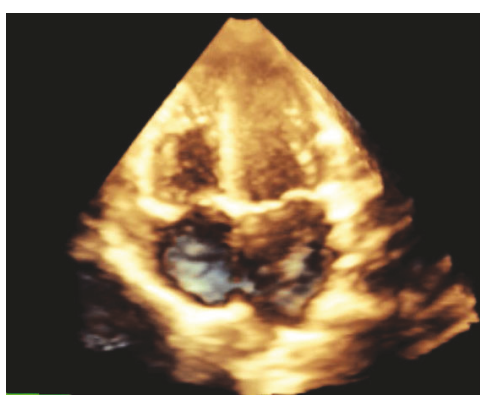

Patient-specific ECHO Images

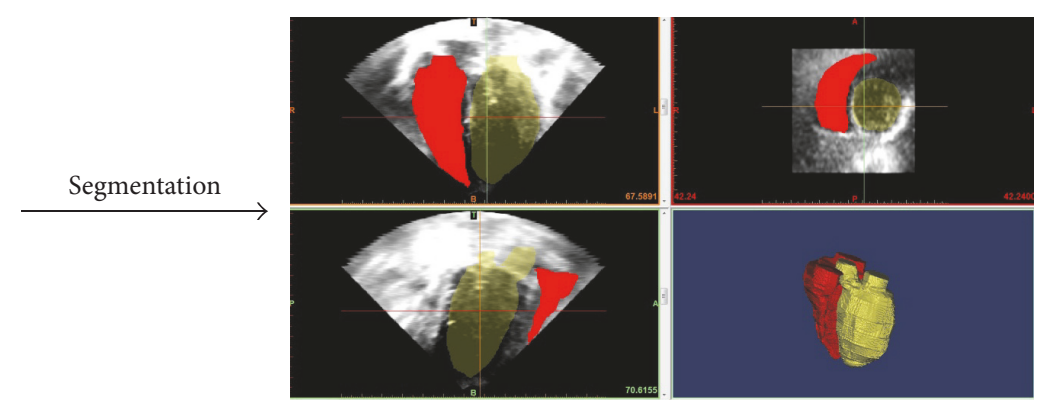

Reconstructed models
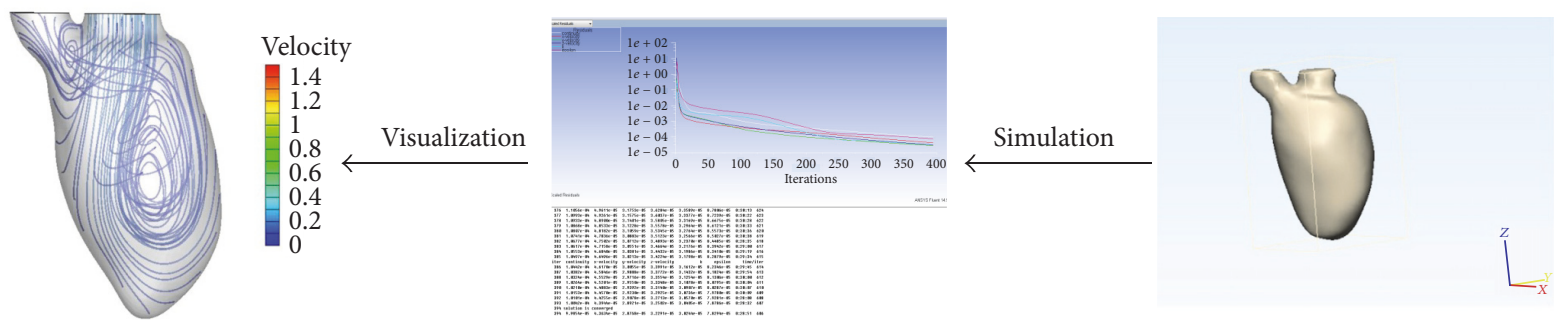

FIGURE 1: The process of CFD 3D model reconstruction and simulation. The CFD modeling is based on real-time 3D echocardiography. A smith dynamic geometry was used to reconstruct the 3D ventricular geometry. The reconstructed model was smoothed by the "Smooth Mask" tool. The reconstructed ventricular geometry along with the boundary conditions were imported into the software (ANSYS ${ }^{\circledR}$-FLUENT) for simulation. Finally, the visualization of the streamlines was completed by the ANSYS CFD-Post 14.5 software.

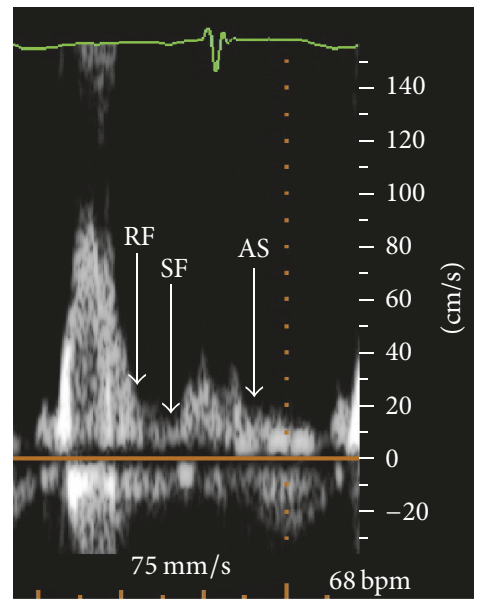

Diastolic flow curve

(a)

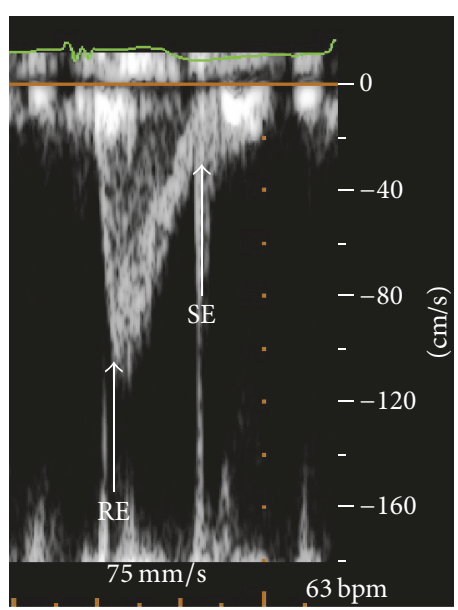

Systolic flow curve

(b)

FIGURE 2: Boundary conditions of the CFD-simulation models from echocardiographic flow curve. The inflow/outflow boundary conditions used for simulation were obtained from the mitral/aortic valve velocities by echocardiography at the end of five characteristic periods. (RF: rapid filling; SF: slow filling; AS: atrial systole; RE: rapid ejection; SE: slow ejection. Arrows are at the end of five periods).

CFD-Post 14.5 software. The SRV patient had one major right atrioventricular valve and one hypoplastic left atrioventricular valve. The patient had only one ventricular outlet with the aortic valve because of pulmonary atresia (Table 2). As described in other simulations of ventricular flow studies [1114], in this CFD model, the dynamic valvular motion was not calculated independently with the ventricular walls; similarly, the endocardium was smoothed in the simulated model. 
TABLE 2: Boundary conditions of the CFD-simulation models (inlet and outlet velocities).

\begin{tabular}{|c|c|c|c|c|c|}
\hline Velocity $(\mathrm{m} / \mathrm{s})$ & PRF & PSF & PAS & PRE & PSE \\
\hline \multicolumn{6}{|l|}{ Control } \\
\hline Inlet $\mathrm{L}$ & 0.21 & 0.14 & 0.20 & 0.00 & 0.00 \\
\hline Outlet L & 0.00 & 0.00 & 0.00 & 1.00 & 0.35 \\
\hline Inlet $\mathrm{R}$ & 0.20 & 0.20 & 0.18 & 0.00 & 0.00 \\
\hline Outlet R & 0.00 & 0.00 & 0.00 & 0.90 & 0.20 \\
\hline \multicolumn{6}{|l|}{ SRV } \\
\hline Inlet L & 0.05 & 0.10 & 0.05 & 0.00 & 0.00 \\
\hline Inlet $\mathrm{R}$ & 0.17 & 0.18 & 0.12 & 0.00 & 0.00 \\
\hline Outlet & 0.00 & 0.00 & 0.00 & 0.89 & 0.38 \\
\hline \multicolumn{6}{|l|}{$\mathrm{SRV}+\mathrm{HF}$} \\
\hline Inlet L & 0.25 & 0.20 & 0.15 & 0.00 & 0.00 \\
\hline Inlet $\mathrm{R}$ & 0.20 & 0.20 & 0.12 & 0.00 & 0.00 \\
\hline Outlet & 0.00 & 0.00 & 0.00 & 0.90 & 0.25 \\
\hline
\end{tabular}

Note. CFD: computational flow dynamics; SRV: single right ventricle without heart failure; SRV + HF: single right ventricle with heart failure; PFR: period of rapid filling; PSF: period of slow filing; PAS: period of atrial systole; PRF: period of rapid ejection; PSE: period of slow ejection; Inlet L: left inlet, mitral valve or left atrioventricular valve level; Inlet R: right inlet, tricuspid valve or right atrioventricular valve level; Outlet L: left outlet, aortic valve level; Outlet R: right outlet, pulmonary valve level.

At isovolumic relaxation, the ventricular muscle continues to relax for about 0.03 to 0.06 seconds, and the intraventricular pressures drop rapidly from about $80 \mathrm{mmHg}$ to almost $0 \mathrm{mmHg}$; then follow the early diastole, with a flow velocity around $1.2 \mathrm{~m} / \mathrm{s}$. The blood in the left ventricle is assumed to be an incompressible Newtonian fluid with a constant viscosity of $0.004 \mathrm{~kg} / \mathrm{ms}$ and density of $1060 \mathrm{~kg} / \mathrm{m}^{3}$. According to the formula of Reynolds index: $\operatorname{Re}=\rho \mathrm{UD} / \mu$, if its Reynolds number $>4000$, it is considered to be turbulent. Therefore, the standard $\kappa-\varepsilon$ model was applied to solve the motion of intraventricular blood flow; this model is a two-equation eddy viscosity model based on the solution of equations for the kinetic energy of turbulence and turbulence dissipation rate. The second-order upwind scheme was employed to complete the steady state numerical simulation by ANSYS-FLUENT 17.0 software. We employed steady state simulation at the three cardiac phases (the end of rapid filling, slow filling, and atrial systole) during diastole to study the cardiac flow status. Finally, boundary conditions from the 2D ECHO data were applied to the finite volume solver package to complete the simulation. When analyzing the vortex during the filling phase, the walls of the models were assumed to be rigid. The blood patterns within the normal RV and SRV were analyzed by the same technique employed for the LV.

During the filling period, the reconstructed and simulated models allowed for the measurements for the dimensions and volume of the ventricles, and the flow dynamics, including maximum length $\left(L_{\mathrm{LV}}, L_{\mathrm{RV}}\right.$ or $\left.L_{\mathrm{DVC}}\right)$, diameter $\left(D_{L V}, D_{\mathrm{RV}}\right.$ or $\left.D_{\mathrm{DVC}}\right)$, and width $\left(W_{\mathrm{LV}}, W_{\mathrm{RV}}\right.$ or $\left.W_{\mathrm{DVC}}\right)$ of the left ventricle (LV), right ventricle $(R V)$, and dominant ventricular chamber (DVC) of the SRV. The ratio of diameter over length of the LV and DVC termed as spherical index (SI) can be derived as well. The area $\left(A_{\mathrm{MV}}, A_{\mathrm{TV}}\right.$, or $\left.A_{\mathrm{AV}}\right)$, perimeter $\left(P_{\mathrm{MV}}, P_{\mathrm{TV}}\right.$, or $\left.P_{\mathrm{AV}}\right)$, and length $\left(L_{\mathrm{MV}}, L_{\mathrm{TV}}\right.$, or $L_{\mathrm{AV}}$ ) of the mitral valve (MV), tricuspid valve (TV), or atrioventricular valve $(\mathrm{AV})$ and the velocity $\left(V_{\mathrm{B}}, V_{\mathrm{M}}, V_{\mathrm{A}}\right.$ or $\left.V_{\mathrm{LB}}, V_{\mathrm{LM}}, V_{\mathrm{LA}}, V_{\mathrm{RB}}, V_{\mathrm{RM}}, V_{\mathrm{RA}}\right)$ of the base, medium, and apex in the LV, RV, and DVC can be acquired as well. In addition, the volumes of the left ventricle $\left(V_{\mathrm{LV}}\right)$, right ventricle $\left(V_{\mathrm{RV}}\right)$, and the whole single right ventricle $\left(V_{\mathrm{SRV}}\right)$ which was calculated as the summary of the volume of the dominant and rudimentary chambers can be achieved. The CFD models also allow the measurements of end-diastolic volume (EDV), end-systolic volume (ESV), stroke volume (SV), and ejection fraction (EF) of normal LV, RV, and SRV; then the body-surface-area standardized EDV, ESV, SV, and EF were calculated. The flow characteristics inside the SRV were examined and compared with those inside the normal LV and RV. Bland-Altman analysis was performed on diameter and velocity measurements to compare intertechnique agreement between CFD-simulated methods and Echo.

\section{Results}

The complexity of the heart anatomy and geometric assumptions of CFD models requires validation of CFD-simulated results, especially in complicated clinical cases. The CFDsimulated results and Echo measured values of the normal subject and the patient were summarized in Tables 3 and 4. The lengths, depths, and widths of the LV, RV, and SRV; SI of the LV and DVC; the areas, perimeters, and lengths of the atrioventricular valve; and the incoming velocities were comparable between the CFD-simulated results and Echo measurements (Figure 3). The qualitative comparisons demonstrated good concordance between the CFD-simulated results and Echo measured values; interclass correlations for diameter and velocity measurements were $0.99(p<0.01)$ and $0.84(p<0.01)$, respectively.

The volumes measured at different phases of the cardiac cycle in normal LV were equivalent to those in the normal RV (Table 5). Similarly, the BSA calibrated volumes and 
TABLE 3: Percentage differences between CFD-simulated values and ECHO measured values in the normal LV and RV.

\begin{tabular}{|c|c|c|c|c|c|c|c|c|c|c|c|}
\hline \multirow{2}{*}{ LV } & \multicolumn{5}{|c|}{ Percentage differences } & \multirow{2}{*}{ RV } & \multicolumn{5}{|c|}{ Percentage differences } \\
\hline & PRF & PSF & PAS & PRE & PSE & & PRF & PSF & PAS & PRE & PSE \\
\hline$L_{\mathrm{LV}}(\mathrm{cm})$ & 1.72 & -4.23 & 0 & 0.47 & 1.5 & $L_{\mathrm{RV}}(\mathrm{cm})$ & 2.09 & 0.60 & -0.18 & -1.00 & -1.35 \\
\hline$D_{\mathrm{LV}}(\mathrm{cm})$ & -5.05 & -4.98 & 1.55 & 3.28 & -1.79 & $D_{\mathrm{RV}}(\mathrm{cm})$ & -8.56 & -3.10 & 0.00 & -2.38 & -2.55 \\
\hline$W_{\mathrm{LV}}(\mathrm{cm})$ & 0 & 4.38 & 9.43 & 2.08 & 14.63 & $W_{\mathrm{RV}}(\mathrm{cm})$ & 1.57 & -3.70 & -0.41 & 0.45 & -0.68 \\
\hline SI & -6.25 & -1.61 & 1.67 & 3.51 & 1.79 & $A_{\mathrm{TV}}\left(\mathrm{cm}^{2}\right)$ & 0.26 & -0.48 & 3.42 & 1.97 & -13.62 \\
\hline$A_{\mathrm{MV}}\left(\mathrm{cm}^{2}\right)$ & 2.01 & -2.35 & -1.16 & 0.45 & -1.47 & $P_{\mathrm{TV}}(\mathrm{cm})$ & 0.14 & -0.13 & 1.58 & -0.26 & -0.14 \\
\hline$P_{\mathrm{MV}}(\mathrm{cm})$ & -0.61 & -2.83 & -2.38 & 0.62 & 1.21 & $L_{\mathrm{TV}}(\mathrm{cm})$ & -2.11 & -2.07 & 3.52 & 0.41 & 3.56 \\
\hline$L_{\mathrm{MV}}(\mathrm{cm})$ & 2.34 & -3.26 & -5.84 & 3.60 & 3.91 & $V_{\mathrm{B}}(\mathrm{m} / \mathrm{s})$ & 18.18 & -11.11 & -25.00 & / & I \\
\hline$V_{\mathrm{B}}(\mathrm{m} / \mathrm{s})$ & 13.33 & 0 & 15.38 & / & I & $V_{\mathrm{M}}(\mathrm{m} / \mathrm{s})$ & 30.00 & -26.67 & -40.00 & / & / \\
\hline$V_{\mathrm{M}}(\mathrm{m} / \mathrm{s})$ & 0 & 0 & 0 & / & I & $V_{\mathrm{A}}(\mathrm{m} / \mathrm{s})$ & 240.00 & 233.33 & 0.00 & / & / \\
\hline$V_{\mathrm{A}}(\mathrm{m} / \mathrm{s})$ & -50 & -60 & 0 & / & I & & & & & & \\
\hline
\end{tabular}

Note. Positive values: CFD-simulated values greater than the ECHO measured values; negative values: $\mathrm{CFD}$-simulated values smaller than the ECHO measured values; CFD: computational flow dynamics; ECHO: echocardiography; LV: left ventricle; PFR: period of rapid filling; PSF: period of slow filing; PAS: period of atrial systole; PRF: period of rapid ejection; PSE: period of slow ejection; $L_{\mathrm{LV}}$ : length of the left ventricle; $D_{\mathrm{LV}}$ : diameter of the left ventricle; $W_{\mathrm{LV}}$ : width of the left ventricle; SI: spherical index; $A_{\mathrm{MV}}$ : area of the mitral valve; $P_{\mathrm{MV}}$ : perimeter of the mitral valve; $L_{\mathrm{MV}}$ : length of the mitral valve; $V_{\mathrm{B}}$ : basal velocity in the left ventricle; $V_{\mathrm{M}}$ : medial velocity in the left ventricle; $V_{\mathrm{A}}$ : apical velocity in the left ventricle. RV: right ventricle; $L_{\mathrm{RV}}$ : length of the right ventricle; $D_{\mathrm{RV}}$ : diameter of the right ventricle; $W_{\mathrm{RV}}$ : width of the right ventricle; $A_{\mathrm{TV}}$ : area of the tricuspid valve; $P_{\mathrm{TV}}$ : perimeter of the tricuspid valve; $L_{\mathrm{TV}}$ : length of the tricuspid valve.

TABLE 4: Percentage differences between CFD-simulated values compared with ECHO measured values in the SRV with and without heart failure (HF).

\begin{tabular}{|c|c|c|c|c|c|c|c|c|c|c|c|}
\hline \multirow{2}{*}{ No HF } & \multicolumn{5}{|c|}{ Simulated values } & \multirow{2}{*}{ HF } & \multicolumn{5}{|c|}{ ECHO measured values } \\
\hline & PRF & PSF & PAS & PRE & PSE & & PRF & PSF & PAS & PRE & PSE \\
\hline$L_{\mathrm{DVC}}(\mathrm{cm})$ & -0.20 & -0.59 & -1.15 & 11.96 & 3.95 & & -1.98 & -7.52 & -11.05 & 2.54 & 0.64 \\
\hline$D_{\text {DVC }}(\mathrm{cm})$ & -4.04 & -1.87 & -4.79 & -0.54 & -8.59 & & 0.00 & -0.47 & -1.61 & 0.82 & 2.84 \\
\hline$W_{\text {DVC }}(\mathrm{cm})$ & -3.37 & -2.33 & -3.09 & 5.85 & -3.23 & & -0.89 & 2.61 & -5.90 & -4.16 & -11.14 \\
\hline SI & -3.53 & -1.19 & -3.26 & -11.90 & -11.84 & & 2.41 & 7.59 & 10.34 & -2.56 & 2.67 \\
\hline$A_{\mathrm{AV}}\left(\mathrm{cm}^{2}\right)$ & -6.46 & -7.43 & 0.39 & 5.94 & 16.13 & & 2.91 & 1.91 & -1.66 & -0.91 & 3.69 \\
\hline$P_{\mathrm{AV}}(\mathrm{cm})$ & -10.99 & -7.42 & -5.22 & -1.19 & -0.89 & & -3.53 & -2.57 & -1.94 & -0.15 & 2.15 \\
\hline$L_{\mathrm{AV}}(\mathrm{cm})$ & -1.00 & 0.75 & -8.45 & -1.49 & -1.11 & & -4.24 & -1.85 & -6.11 & -2.64 & 0.25 \\
\hline$V_{\mathrm{LB}}(\mathrm{m} / \mathrm{s})$ & -60.00 & 20.00 & -20.00 & I & I & & 9.09 & 0.00 & -80.00 & / & I \\
\hline$V_{\mathrm{LM}}(\mathrm{m} / \mathrm{s})$ & -12.50 & 0.00 & 0.00 & I & I & & -70.00 & -25.00 & -40.00 & I & I \\
\hline$V_{\mathrm{LA}}(\mathrm{m} / \mathrm{s})$ & 0.00 & 60.00 & 0.00 & I & / & & -50.00 & 75.00 & 25.00 & / & I \\
\hline$V_{\mathrm{RB}}(\mathrm{m} / \mathrm{s})$ & 0.00 & 0.00 & 14.29 & I & I & & -20.00 & 22.73 & -9.09 & / & I \\
\hline$V_{\mathrm{RM}}(\mathrm{m} / \mathrm{s})$ & -16.67 & 0.00 & 77.78 & I & I & & -20.00 & 20.00 & -27.27 & / & I \\
\hline$V_{\mathrm{RA}}(\mathrm{m} / \mathrm{s})$ & 0.00 & -27.27 & 66.67 & I & I & & -20.00 & 5.00 & -12.50 & I & I \\
\hline
\end{tabular}

Note. Positive values: CFD-simulated values greater than the ECHO measured values; negative values: $\mathrm{CFD}$-simulated values smaller than the ECHO measured values; CFD: computational flow dynamics; ECHO: echocardiography; SRV: single right ventricle; PFR: period of rapid filling; PSF: period of slow filing; PAS: period of atrial systole; PRF: period of rapid ejection; PSE: period of slow ejection; $L_{\mathrm{DVC}}$ : length of the dominate ventricular chamber; $D_{\mathrm{DVC}}$ : diameter of the dominate ventricular chamber; $W_{\mathrm{DVC}}$ : width of the dominate ventricular chamber; SI: spherical index; $A_{\mathrm{AV}}$ : area of the atrioventricular valve; $P_{\mathrm{AV}}$ : perimeter of the atrioventricular valve; $L_{\mathrm{AV}}$ : length of the atrioventricular valve; $V_{\mathrm{LB}}$ : basal velocity of the left atrioventricular valve; $V_{\mathrm{LM}}$ : medial velocity of the left atrioventricular valve; $V_{\mathrm{LA}}$ : apical velocity of the left atrioventricular valve; $V_{\mathrm{RB}}$ : basal velocity of the right atrioventricular valve; $V_{\mathrm{RM}}$ : medial velocity of the right atrioventricular valve; $V_{\mathrm{RA}}$ : apical velocity of the right atrioventricular valve.

EF were interchangeable between the normal LV and RV (Table 6). The volumes of the SRV were larger than those of normal LV and RV and much larger when heart failure occurred. The volumes measured at different phases of the cardiac cycle in the SRV demonstrated significant increase when heart failure was present (Table 5). In addition, the BSA calibrated volumes and EF illustrated the impaired functions in SRV when heart failure was present (Table 6). The EF of the SRV without heart failure was comparable to that of normal LV and RV but significantly decreased while suffering heart failure. Spherical index (SI) is the ratio between the short and long axis length, and spherical deformation is the transformation of the ventricle into a spherical shape with an increased circumference relative to the longitudinal deformation. Due to triangular/crescent shape of the normal $\mathrm{RV}$, SI of the RV was not applicable and was not calculated. SI of the LV and the SRV increased during diastole, reached their maximum during atrial systole, and then decreased and 


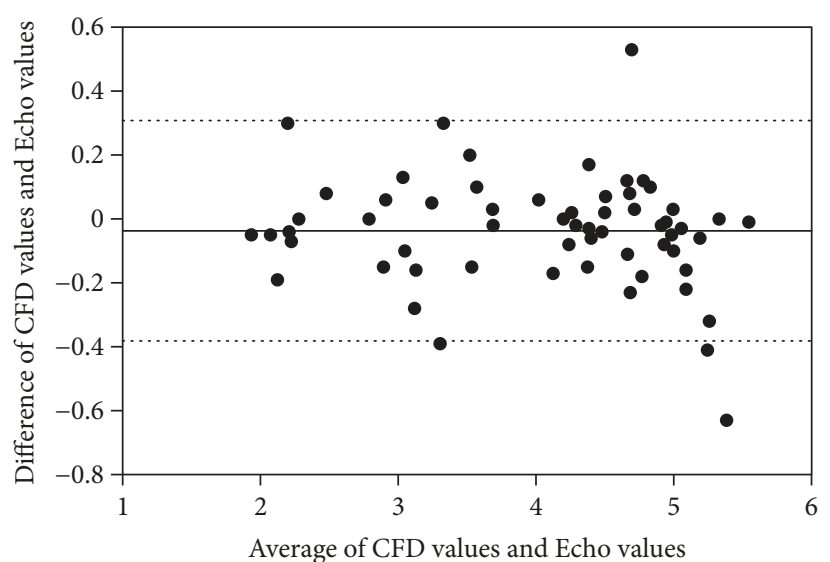

(a)

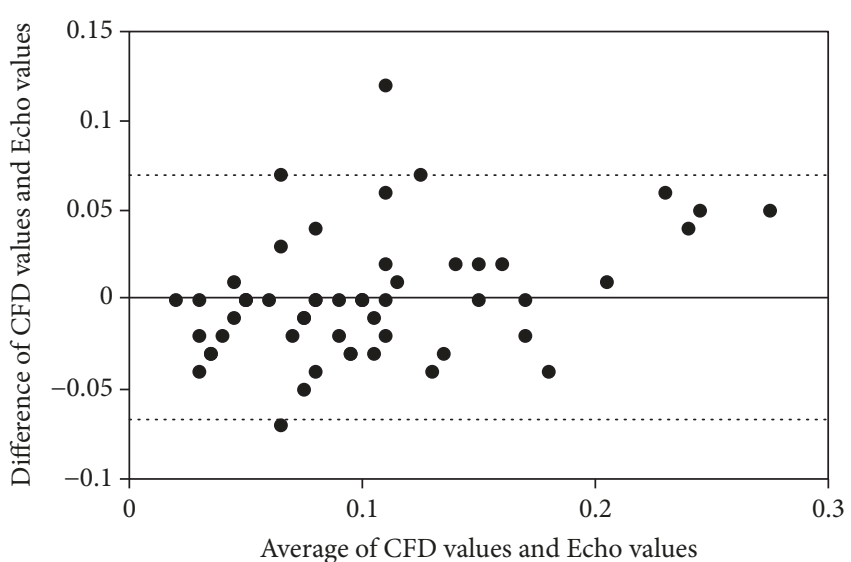

(b)

FIGURE 3: Bland-Altman analysis of diameter and velocity between CFD and echocardiography measurements. ((a) Bland-Altman analysis of diameter measurements; (b) Bland-Altman analysis of velocity measurements).

TABLE 5: CFD derived volume measurements of the LV, RV, and SRV.

\begin{tabular}{lccccc}
\hline Volume $(\mathrm{ml})$ & PRF & PSF & PAS & PRE & PSE \\
\hline Control & & & & & \\
$\quad V_{\text {LV }}$ & 24.20 & 27.10 & 39.01 & 23.81 & 15.50 \\
$\quad V_{\text {RV }}$ & 28.11 & 29.37 & 40.25 & 26.41 & 16.74 \\
SRV & & & & & \\
$\quad V_{\text {SV }}$ & 48.35 & 50.53 & 63.86 & 37.98 & 27.56 \\
$\quad$ SRV + HF & & & & & \\
$\quad V_{\text {SV }}$ & 51.96 & 56.06 & 68.64 & 49.54 & 42.38 \\
\hline
\end{tabular}

Note. CFD: computational flow dynamics; LV: left ventricle; RV: right ventricle; SRV: single right ventricle without heart failure; SRV + HF: single right ventricle with heart failure; PFR: period of rapid filling; PSF: period of slow filing; PAS: period of atrial systole; PRF: period of rapid ejection; PSE: period of slow ejection; $V_{\mathrm{LV}}$ : volume of the left ventricle; $V_{\mathrm{RV}}$ : volume of the right ventricle; $V_{\mathrm{SV}}$ : volume of the whole functional single ventricle.

TABLE 6: BSA calibrated volume and EF of the normal child and the patient.

\begin{tabular}{lcccc}
\hline & $\mathrm{EDV}\left(\mathrm{ml} / \mathrm{m}^{2}\right)$ & $\mathrm{ESV}\left(\mathrm{ml} / \mathrm{m}^{2}\right)$ & $\mathrm{SV}\left(\mathrm{ml} / \mathrm{m}^{2}\right)$ & $\mathrm{EF}(\%)$ \\
\hline Control (LV) & 60.01 & 23.85 & 36.16 & 60.26 \\
Control (RV) & 61.92 & 25.75 & 36.17 & 58.41 \\
SRV & 98.25 & 42.40 & 55.85 & 56.84 \\
SRV + HF & 91.52 & 56.51 & 35.01 & 38.25 \\
\hline
\end{tabular}

Note. BSA: body surface area; SRV: single right ventricle without heart failure; SRV + HF: single right ventricle with heart failure; LV: left ventricle; RV: right ventricle; EDV: end-diastolic volume; ESV: end-systolic volume; SV: stroke volume; EF: ejection fraction.

reached their minimum during the period of slow ejection. SI of the normal LV during atrial systole is 0.61 , whereas those of the SRV, with and without heart failure, were 0.89 and 0.96 , respectively, much greater than normal values (Tables 3 and 4).

CFD enables visualization of the dynamic flow by demonstrating the streamline in the heart, in which the direction and magnitude (color) of the flow can be determined. In normal left ventricle, during the period of rapid filling, blood flew through the mitral valve reaching the apex and swirling, forming a major vortex in the middle of the left ventricle (Figure 4). The major vortex exhibited counterclockwise rotation and persisted throughout the entire diastolic period. During the atrial systolic phase, the vortex was enlarged, occupying almost the entire ventricle. A small clockwise rotation vortex was present at the outflow tract region. A vortex ring was observed in the base region downstream of the inlet orifice (Figure 5).

In normal right ventricle during diastole, the blood went helical and flowed from the tricuspid region toward the free wall and the septal region. The vortex was clockwise and presented at the junction of the tricuspid valve and pulmonary valve; it was larger in size than the vortex inside the LV (Figure 4). Rotating flow was observed near the tricuspid valve, elongating and forming a vortex ring toward the outlet orifice. The RV vortex ring had similar orientation as the LV vortex ring, but with different circularity; it was not as annular as the corresponding $L V$ vortex from vertical view (Figure 5).

In the SRV without heart failure, during the period of rapid filling, blood flew through the atrioventricular valve and entered the dominant chamber; part of the flow streamed straight ahead to the apex, and part of the flow moved toward the posterior wall to form a vortex near the bulboventricular foramen. The vortex persisted until the slow filling phase and moved toward the interior of the rudimentary chamber. During the atrial systolic phase, a vortex appeared at the aortic root. The vortices in the SRV without heart failure were smaller than those in the normal LV and RV (Figure 6), and no vortex ring was observed in the SRV during diastole.

The vortices changed in the SRV when heart failure was present (Figure 7): there was only one small vortex near the bulboventricular foramen during the rapid filling phase, with rambling streamlines during other diastolic phases. The vortex was smaller than that in SRV without heart failure 


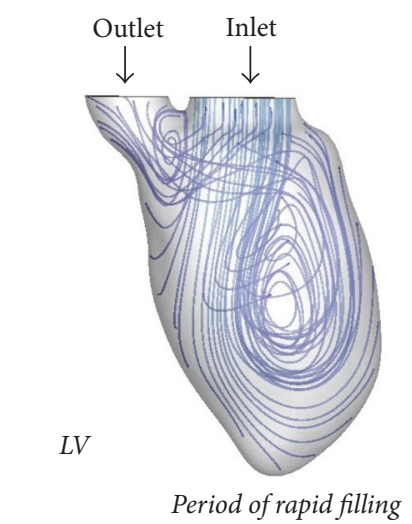

(a)

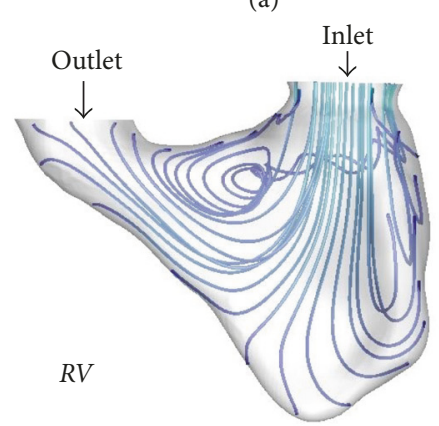

Period of rapid filling

(d)

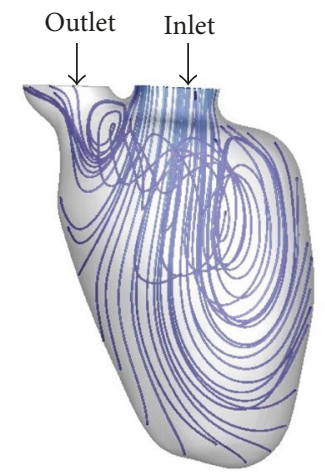

Period of slow filling

(b)

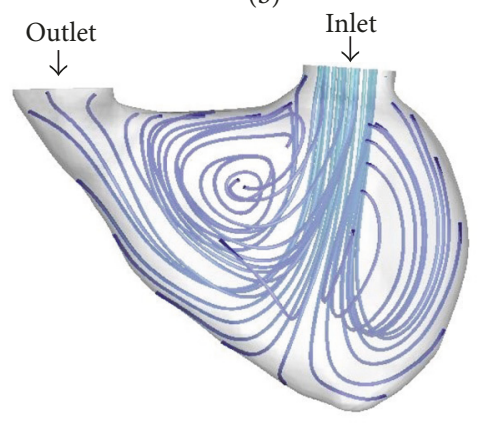

Period of slow filling

(e)

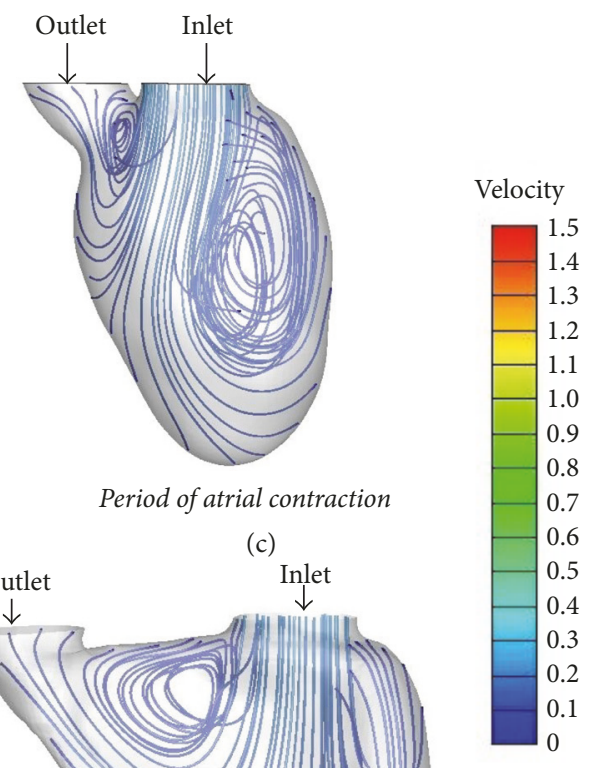

Period of atrial contraction

(f)

FIGURE 4: Streamline in the normal $L V$ and $R V$ during diastole $((\mathrm{a}-\mathrm{c})$ streamlines in the LV during the rapid filling, slow filling, and atrial systole period; (d-f) streamlines in the RV during the rapid filling, slow filling, and atrial systole period).

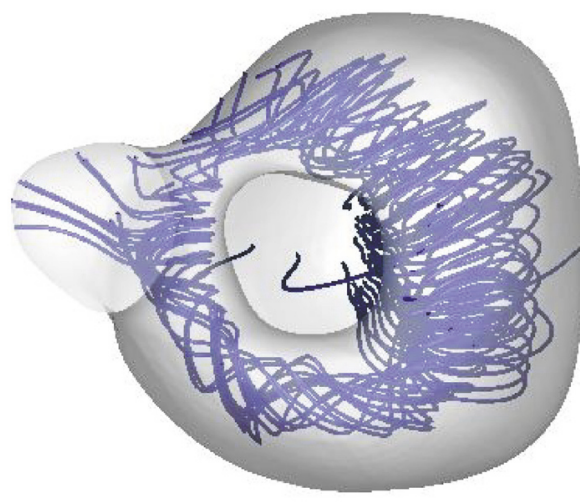

(a)

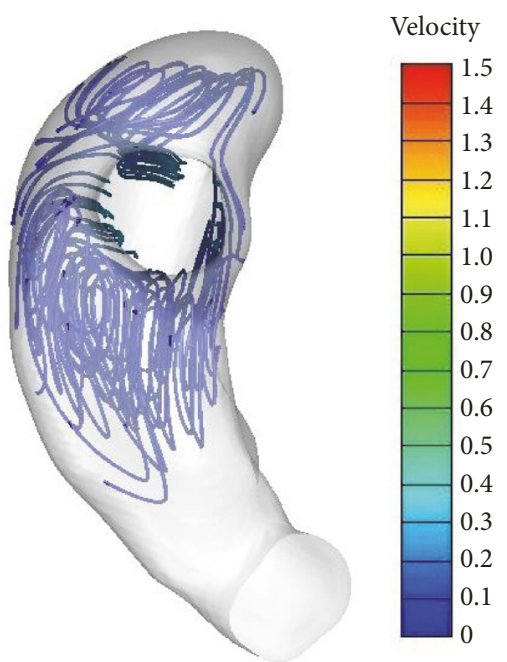

(b)

FIGURE 5: Vortex rings in the normal $L V$ and RV during diastole ((a) vertical view of the LV; (b) vertical view of the RV). 


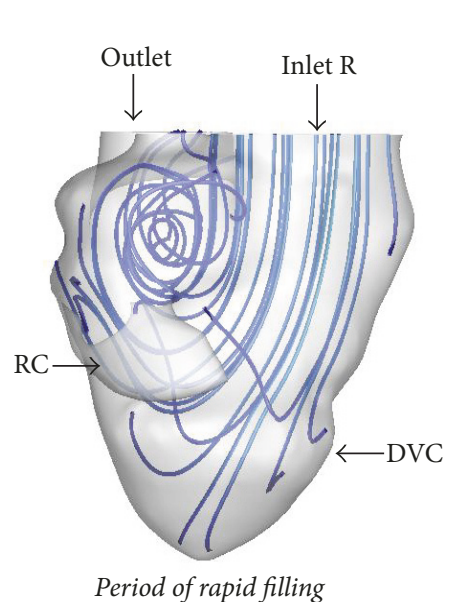

(a)

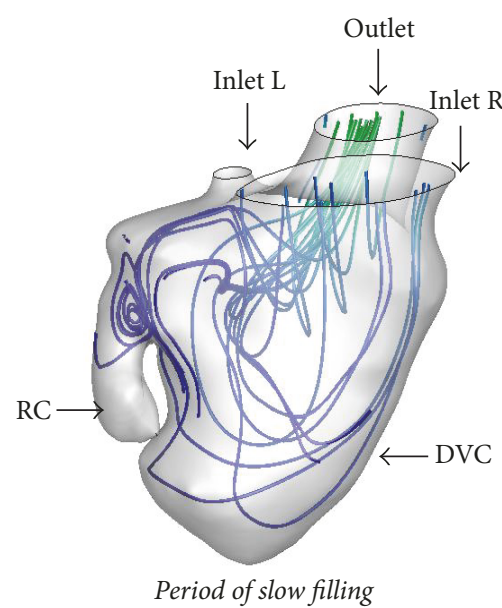

(b)

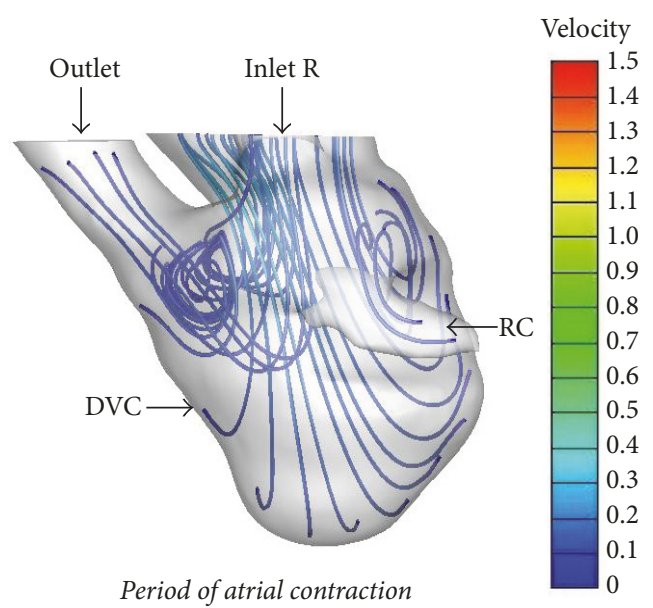

(c)

FIGURE 6: Streamline in the SRV without heart failure during diastole ((a) period of rapid filling; (b) period of slow filling; (c) period of atrial systole). DVC: dominant ventricular chamber; RC: rudimentary chamber.

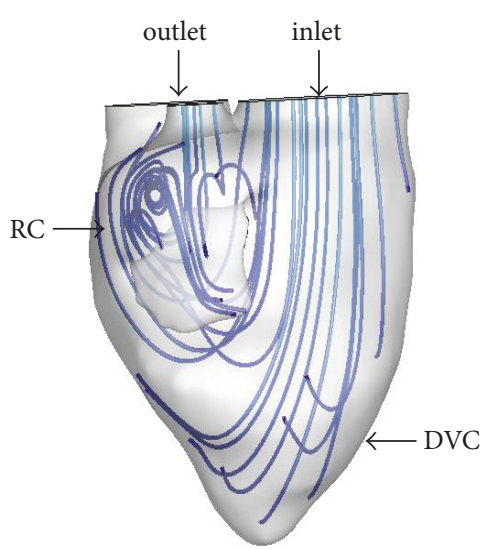

Period of rapid filling

(a)

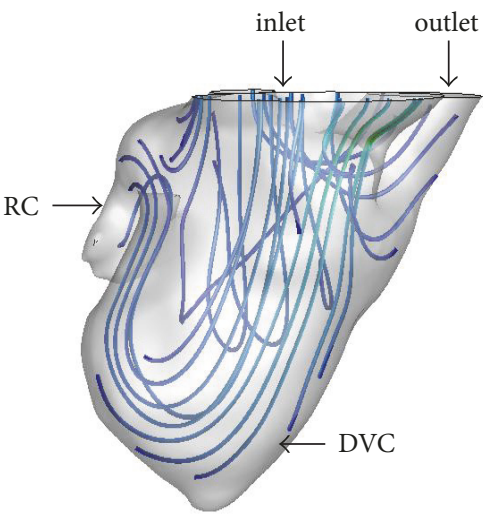

Period of slow filling

(b)

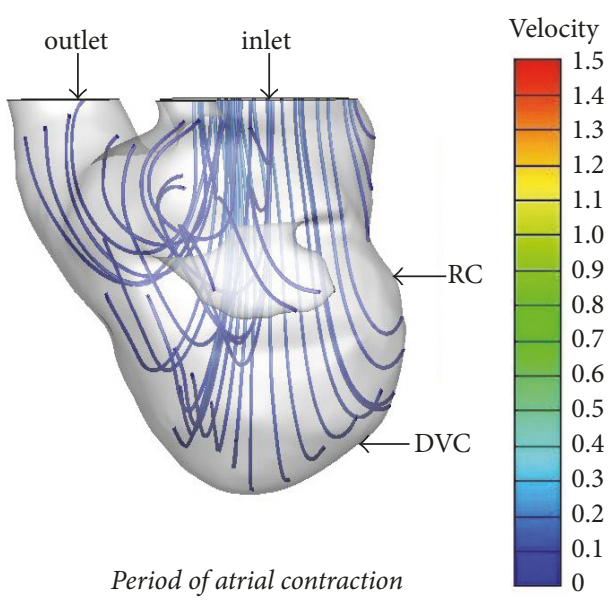

(c)

FIGURE 7: Streamline in the SRV with heart failure during diastole. ((a) period of rapid filling; (b) period of slow filling; (c) period of atrial systole). DVC: dominant ventricular chamber; RC: rudimentary chamber.

and normal LV and RV; furthermore, the vortex disappeared during the slow filling and atrial systole period. No vortex ring was observed during diastole in SRV with or without heart failure.

Turbulent kinetic energy (TKE) was analyzed in the LV and RV and in the SRV with/without heart failure (Figure 8). In normal LV and RV, the TKE values were close to zero and evenly distributed in the ventricle, while, in the SRV, the TKE showed a larger gradient dispersion throughout the ventricle.

\section{Discussion}

This study applied CFD derived 3D numerical simulations to measure the dimensions, volumes, and functions of ventricles and to visualize the flow dynamics inside of normal LV, RV, and SRV. The qualitative comparisons of dimensions, volumes, and functions showed good agreements between the CFD-simulated results and Echo measured values (Figure 3), which manifested and validated the feasibility and reliability of CFD in monitoring blood flow, geometry, and function of single right ventricle.

Ventricular dysfunction is an important risk factor for the morbidity and mortality in patients with a single ventricle [15]. Blood flow pattern inside the ventricular chamber is related to the functional and morphologic changes of the heart [16]; flow pattern even changes before overt changes occurred in cardiac function.

Previous studies have confirmed that the natural vortex motion in the healthy LV may be corrupted when dysfunctions occur [17]. Liang et al. reported the development of 


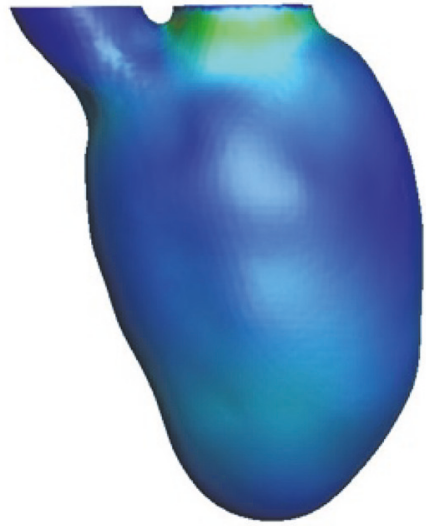

LV

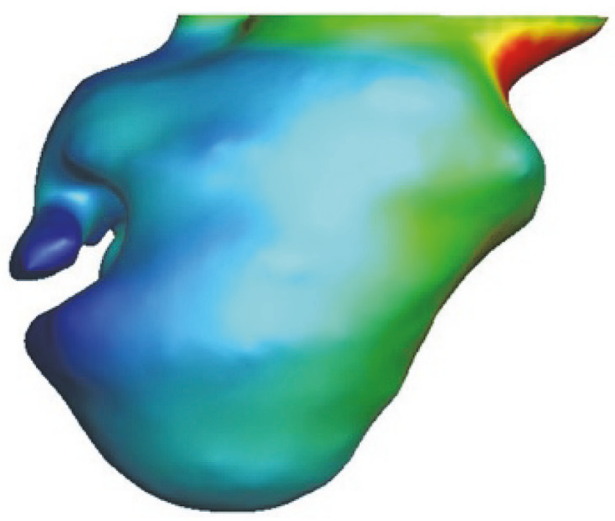

SRV without heart failure
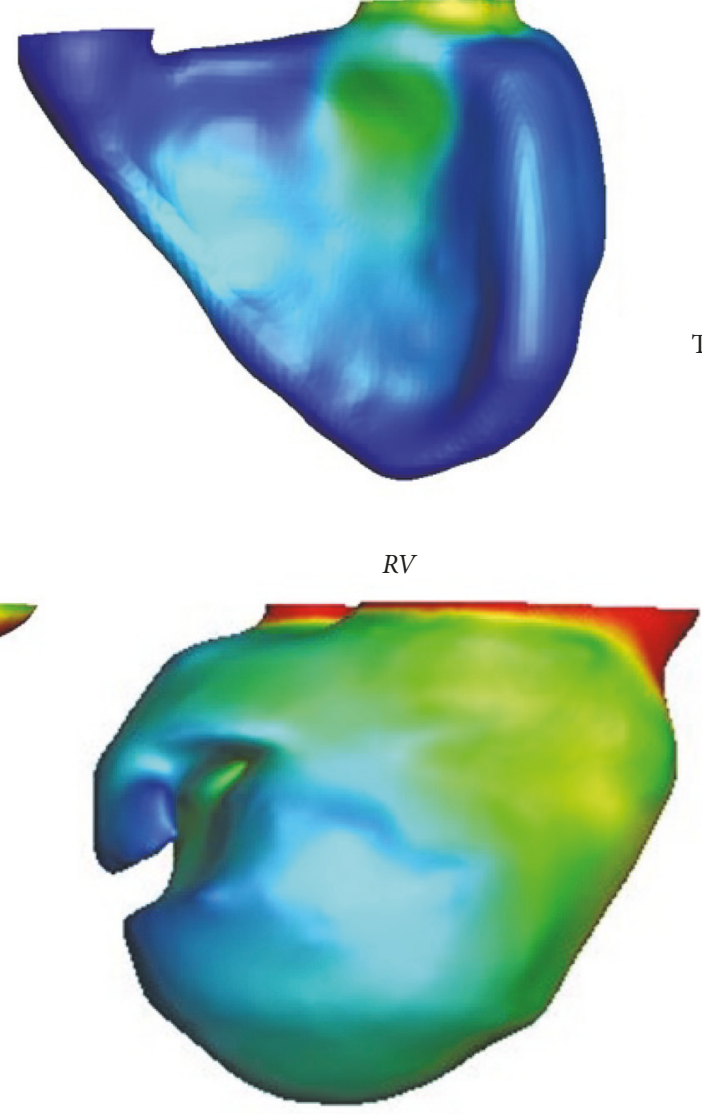

SRV with heart failure
Turbulent kinetic energy

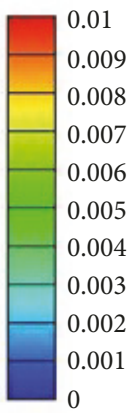

FIGURE 8: Turbulent Kinetic Energy (TKE) of LV, RV, and SRV during period of atrial systole. In normal LV and RV, the TKE values were close to zero; it is concentric and evenly distributed in the ventricle; however, in the SRV, the TKE showed a larger gradient dispersion throughout the ventricle.

a spherical LV shape accompanying heart failure leading to pronounced deterioration of the pump function of the LV [18]. Therefore, analyzing blood flow dynamics has incremental values in early detection of ventricular dysfunction. The formation of transmitral vortices may be a sensitive marker of diastolic dysfunction [19]. Previous studies [17, 2022] demonstrated blood flow swirling and forming vortices during the rapid filling phase, which were introduced by both the geometry of the left ventricle and the curvature between the mitral valve and the aorta. Blood flowing through the mitral valve that reaches the apex of the heart would form a major vortex in the middle of the left ventricle. This vortex exhibits counterclockwise rotation and persists throughout diastole. Another clockwise rotation vortex is present near the outflow tract; the formation of this vortex may be due to the expansion of a large cross-sectional area at the junction of the inlet and outlet, which separates the flow. A vortex ring forms near the mitral orifice region, redirecting the flow and preserving the energy. Ventricular relaxation during diastole allows the myocardium to recoil and pump blood into the ventricle. The size and shape of the ventricle may affect the turning of streamlines and the formation of the vortices.
Similar to previous reports $[17,23]$, we observed that the early inflow goes toward the free wall and the septal region in the normal right ventricle during diastole. A larger clockwise vortex was observed at the junction of the tricuspid valve and pulmonary valve. The formation of this vortex may be due to the funnel shape of RV and the expansion of a large crosssectional area at the junction of the inlet and outlet which separates the flow. A vortex ring presented near the tricuspid region and elongated to form the helical spinning flow toward the pulmonary orifice. The RV vortex ring was less annular when compared with the LV vortex ring due to the special triangular shape of the RV. Presence of vortex ring may be related to the low pressure gradient inside the right ventricle during diastole. The funnel-like RV geometry assists the flow for ejection by converging toward the pulmonary orifice [5]. Diastolic flow in normal RV makes it more favorable for effective systolic ejection [24].

Myocardial relaxation and contraction are the dynamic energy sources of intracardiac flow. Vortices offer the most appropriate spatial pressure distribution to push the flow. It can impound a certain amount of flow energy during diastolic phase, converting vortex kinetic energy into the rotational 
kinetic energy by means of gyratory motion, which averts a strong convective deceleration. The diastolic vortices can redirect the flow to avoid excessive dissipation of energy, thereby facilitating ventricular filling and stroke volume maintenance [25]. The natural asymmetric geometry of heart could minimize dissipative interaction of flow convection to arrange the flow for ejection, while unnatural asymmetry flow could reduce the efficiency of the heart pump by more than $10 \%$ [26]. Normal flow pattern inside the ventricle is altered in dilated and hypocontractile hearts, accompanied with weakened pumping efficiency [26]. The sphericity of the ventricle makes the atrioventricular annulus more centered or highly eccentric, which may increase dissipation, as reported by Pedrizzetti and Domenichini [26]. It was observed in our study that the turbulent kinetic energy in the SRV was dispersed in the ventricle; however it is more centered and evenly distributed in the normal LV and RV. The abnormal geometry of SRV contributed to the increased dissipation and energy loss of blood flow.

According to previous studies, SRV have reduced systolic and diastolic function compared with those of the respective ventricles in normal biventricular hearts [1]. The SRV has a dominant RV ventricle and a second, hypoplastic rudimentary LV chamber. The main ventricular chamber has a RV anatomy but enlarged to a spherical shape. The morphology of the SRV may be less suitable for the systemic circulation than left ventricle. The vortex during diastole in patient with SRV was much smaller than those in normal LV and RV. When heart failure is present, the vortex inside the ventricle may diminish or even disappear. The myocardial adaptation to chronic systemic pressure has been demonstrated via the transformation of the ventricle into a spherical shape for more efficient contraction [27]. Increased end-systolic chamber size induces atrioventricular valve disproportion with weakening of the vortex. The diminished or obliterated diastolic vortex may increase ventricular work because of decreased energy storage due to the morphologic abnormality of the right ventricle. When natural arrangement is broken, energy dissipation increases, possibly ejecting less energetic blood into the ventricular outlet tract. This reaction may have the consequence of increasing mechanical work of the cardiac muscle to maintain equal energy and eject sufficient amount of flow into the systemic circulation. As a result, it cannot afford the work load; therefore its ventricular myocardium decompensation would result in extra energy dissipation, and ultimately heart dysfunction. Kaneko et al. suggested that the single ventricle with dominant right ventricular morphology may be associated with impaired systolic and diastolic functional parameters [28]; SRV has impaired ventricular function and abnormal flow structure. Smaller or obliterated vortices may prompt the deterioration of ventricular function. CFD may provide a new way of unmasking the myocardium dysfunction in SRV by detecting early changes in the number and shape of the vortices in the ventricle.

To our knowledge, this is the first report of using CFD to evaluate the flow pattern in SRV, but there are some limitations of this study. (1) The CFD analysis in this study was based on echocardiographic images; it suffers the same spatial and temporal resolutions limitations of echocardiography. (2) The SRV papillary muscles and valves were smoothing out to optimize CFD modeling. In spite of its limitations, CFD still can provide detailed real-time information about complex intraventricular flow pattern, leading to a better understanding of both physiological and pathological hemodynamics in SRV.

\section{Conclusion}

In summary, CFD 3D modeling can not only evaluate the morphological and functional abnormalities in SRV, but also detect the abnormal flow pattern and vortices during diastole. CFD shows promising role as an additional noninvasive tool to assist conventional clinical techniques in visualizing flow in the heart as well as gain further understanding and optimize their treatment.

\section{Disclosure}

All of the authors take full responsibility for all aspects of the reliability and freedom from bias of the data presented, and their discussed interpretation.

\section{Conflicts of Interest}

The authors declare no competing financial interests.

\section{Acknowledgments}

The authors express their great gratitude for the support of the National Nature Science Foundation of China [Grant no. 81501558], the Project sponsored by the Scientific Research Foundation for the Returned Overseas Chinese Scholars, State Education Ministry [Grant no. 20144902], the Programs of the Shanghai Science and Technology Commission [Grant no. 14411968900], the Biomedical and Engineering (Science) Interdisciplinary Study Fund of Shanghai Jiaotong University [Grant no. YG2014MS63], the Project funded by China Postdoctoral Science Foundation [Grant no. 2014T70420], the Programs of the Shanghai Science and Technology Commission [Grant no. 14411961700], an Innovation Program of the Shanghai Municipal Education Commission [Grant no. 13ZZ081], the Fund of Shanghai Jiaotong University School of Medicine [Grant no. 14XJ10039], and the Clinical Science and Technology Innovation Project of Shanghai Shen Kang Hospital Development Center [Grant no. SHDC22015019].

\section{References}

[1] Y.-R. Wu, Y.-Q. Zhang, L.-J. Chen, S.-S. Wang, S.-W. Zhong, and Z.-F. Zhang, "Assessment of longitudinal systolic ventricular dysfunction and asynchrony using velocity vector imaging in children with a single right ventricle," Pediatric Cardiology, pp. 1147-1154, 2014.

[2] K. Isaaz and A. Pasipoularides, "Noninvasive assessment of intrinsic ventricular load dynamics in dilated cardiomyopathy," Journal of the American College of Cardiology, vol. 17, no. 1, pp. 112-121, 1991. 
[3] W. J. Paulus, D. L. Brutsaert, T. C. Gillebert et al., "How to diagnose diastolic heart failure," European Heart Journal, vol. 19, no. 7, pp. 990-1003, 1998.

[4] B. Baccani, F. Domenichini, and G. Pedrizzetti, "Vortex dynamics in a model left ventricle during filling," European Journal of Mechanics - B/Fluids, vol. 21, no. 5, pp. 527-543, 2002.

[5] J. O. Mangual, F. Domenichini, and G. Pedrizzetti, “Describing the highly three dimensional right ventricle flow," Annals of Biomedical Engineering, vol. 40, no. 8, pp. 1790-1801, 2012.

[6] D. R. Muñoz, M. Markl, J. L. M. Mur et al., "Intracardiac flow visualization: Current status and future directions," European Heart Journal-Cardiovascular Imaging, vol. 14, no. 11, pp.10291038, 2013.

[7] H. Zhang, L. Liu, L. Chen et al., "The evolution of intraventricular vortex during ejection studied by using vector flow mapping," Journal of Echocardiography, vol. 30, no. 1, pp. 27-36, 2013.

[8] L. Agati, S. Cimino, G. Tonti et al., "Quantitative analysis of intraventricular blood flow dynamics by echocardiographic particle image velocimetry in patients with acutemyocardial infarction at different stages of left ventricular dysfunction," European Heart Journal_Cardiovascular Imaging, vol. 15, no. 11, pp. 1203-1212, 2014.

[9] J. F. LaDisa Jr., R. J. Dholakia, C. A. Figueroa et al., "Computational simulations demonstrate altered wall shear stress in aortic coarctation patients treated by resection with end-to-end anastomosis," Congenital Heart Disease, vol. 6, no. 5, pp. 432443, 2011.

[10] S. S. Khalafvand, E. Y. K. Ng, and L. Zhong, "CFD simulation of flow through heart: a perspective review," Computer Methods in Biomechanics and Biomedical Engineering, vol. 14, no. 1, pp. 113-132, 2011.

[11] F. Domenichini, G. Querzoli, A. Cenedese, and G. Pedrizzetti, "Combined experimental and numerical analysis of the flow structure into the left ventricle," Journal of Biomechanics, vol. 40, no. 9, pp. 1988-1994, 2007.

[12] T. Doenst, K. Spiegel, M. Reik et al., "Fluid-Dynamic Modeling of the Human Left Ventricle: Methodology and Application to Surgical Ventricular Reconstruction," The Annals of Thoracic Surgery, vol. 87, no. 4, pp. 1187-1195, 2009.

[13] N. R. Saber, N. B. Wood, A. D. Gosman et al., "Progress towards patient-specific computational flow modeling of the left heart via combination of magnetic resonance imaging with computational fluid dynamics," Annals of Biomedical Engineering, vol. 31, no. 1, pp. 42-52, 2003.

[14] H. Watanabe, S. Sugiura, H. Kafuku, and T. Hisada, "Multiphysics simulation of left ventricular filling dynamics using fluid-structure interaction finite element method," Biophysical Journal, vol. 87, no. 3, pp. 2074-2085, 2004.

[15] S. C. Menon, J. A. Dearani, and F. Cetta, "Long-term outcome after atrioventricular valve surgery following modified Fontan operation," Cardiology in the Young, vol. 21, no. 1, pp. 83-88, 2011.

[16] G. Reiter, U. Reiter, G. Kovacs et al., "Magnetic resonancederived 3-dimensional blood flow patterns in the main pulmonary artery as a marker of pulmonary hypertension and a measure of elevated mean pulmonary arterial pressure.," Circulation: Cardiovascular Imaging, vol. 1, no. 1, pp. 23-30, 2008.

[17] M. Markl, P. J. Kilner, and T. Ebbers, "Comprehensive 4D velocity mapping of the heart and great vessels by cardiovascular magnetic resonance," Journal of Cardiovascular Magnetic Resonance, vol. 13, no. 1, article no. 7, 2011.
[18] F. Liang, H. Taniguchi, and H. Liu, "A multi-scale computational method applied to the quantitative evaluation of the left ventricular function," Computers in Biology and Medicine, vol. 37, no. 5, pp. 700-715, 2007.

[19] P. P. Sengupta, G. Pedrizzetti, P. J. Kilner et al., "Emerging trends in CV flow visualization," JACC: Cardiovascular Imaging, vol. 5, no. 3, pp. 305-316, 2012.

[20] S. S. Khalafvand, E. Y. K. Ng, L. Zhong, and T. K. Hung, "Fluiddynamics modelling of the human left ventricle with dynamic mesh for normal and myocardial infarction: preliminary study," Computers in Biology and Medicine, vol. 42, no. 8, pp. 863-870, 2012.

[21] Y. Cheng, H. Oertel, and T. Schenkel, "Fluid-structure coupled CFD simulation of the left ventricular flow during filling phase," Annals of Biomedical Engineering, vol. 33, no. 5, pp. 567-576, 2005.

[22] B. Su, L. Zhong, X.-K. Wang et al., "Numerical simulation of patient-specific left ventricular model with both mitral and aortic valves by FSI approach," Computer Methods and Programs in Biomedicine, vol. 113, no. 2, pp. 474-482, 2014.

[23] M. S. ElBaz, E. Calkoen, J. J. Westenberg, B. P. Lelieveldt, A. Roest, and R. J. van der Geest, "Three dimensional right ventricular diastolic vortex rings: characterization and comparison with left ventricular diastolic vortex rings from 4D flow MRI," Journal of Cardiovascular Magnetic Resonance, vol. 16, no. 1, p. 42, 2014.

[24] C. Carlhäll, A. G. Fredriksson, J. Zajac et al., "Visualization and quantification of $4 \mathrm{D}$ blood flow distribution and energetics in the right ventricle," Journal of Cardiovascular Magnetic Resonance, vol. 13, no. 1, p. O83, 2011.

[25] A. Pasipoularides, "Evaluation of right and left ventricular diastolic filling," Journal of Cardiovascular Translational Research, vol. 6, no. 4, pp. 623-639, 2013.

[26] G. Pedrizzetti and F. Domenichini, "Nature optimizes the swirling flow in the human left ventricle," Physical Review Letters, vol. 95, no. 10, Article ID 108101, 2005.

[27] N. S. Khoo, J. F. Smallhorn, S. Kaneko, K. Myers, S. Kutty, and E. B. Tham, "Novel insights into RV adaptation and function in hypoplastic left heart syndrome between the first 2 stages of surgical palliation," JACC: Cardiovascular Imaging, vol. 4, no. 2, pp. 128-137, 2011.

[28] S. Kaneko, N. S. Khoo, J. F. Smallhorn, and E. B. Tham, "Single right ventricles have impaired systolic and diastolic function compared to those of left ventricular morphology," Journal of the American Society of Echocardiography, vol. 25, no. 11, pp. 12221230, 2012. 


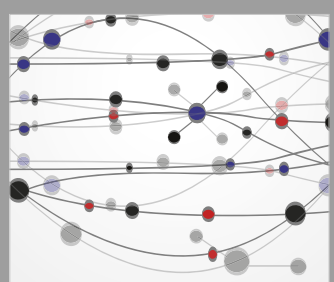

The Scientific World Journal
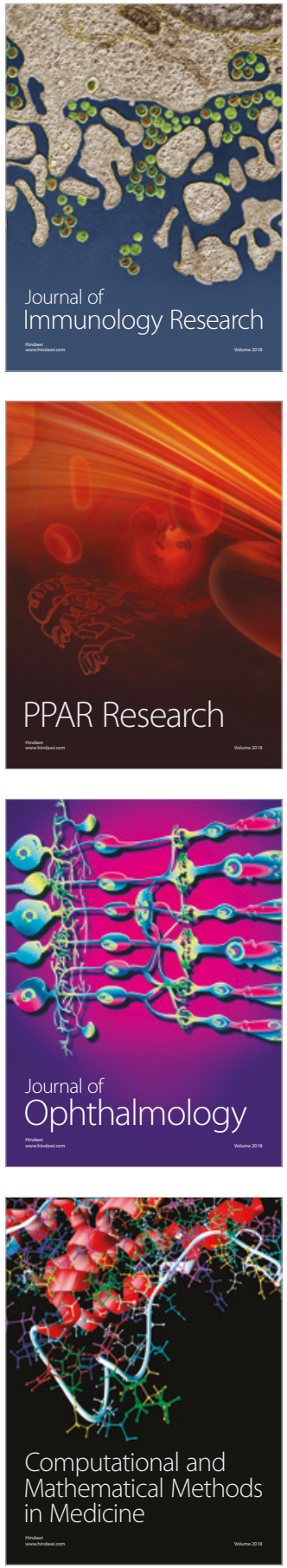

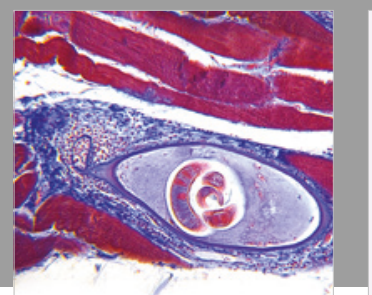

Gastroenterology Research and Practice

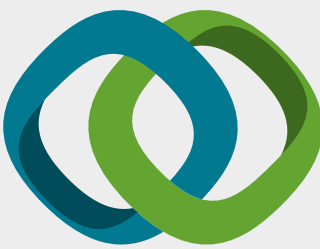

\section{Hindawi}

Submit your manuscripts at

www.hindawi.com
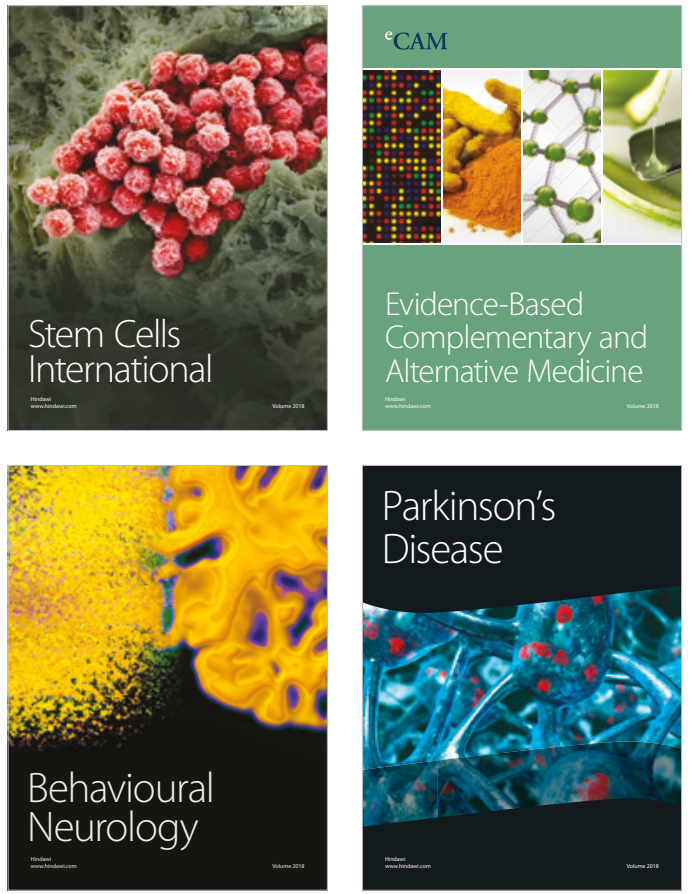

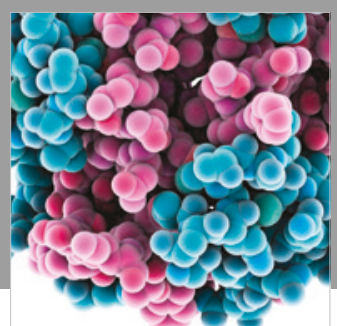

ournal of

Diabetes Research

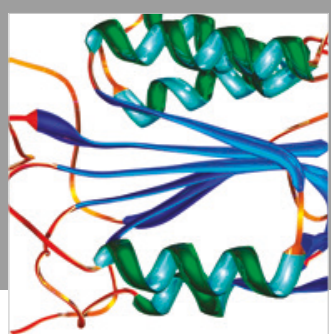

Disease Markers
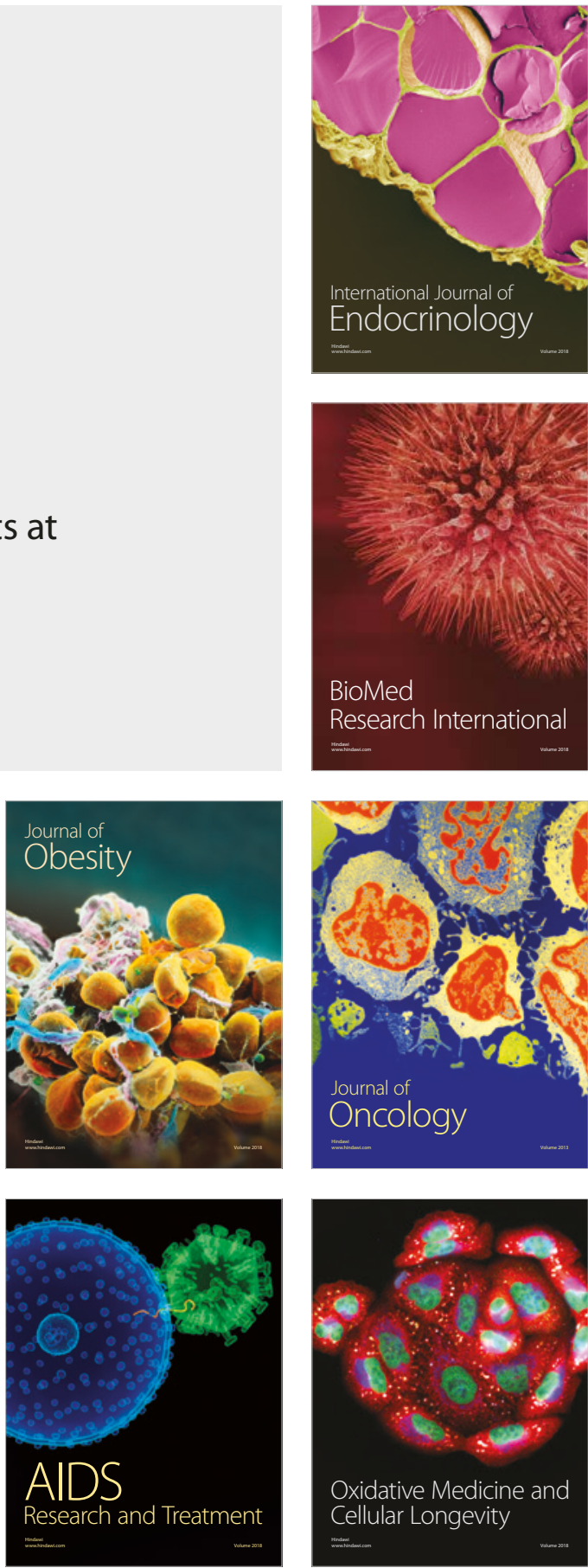